\title{
Analogical scaffolding and the learning of abstract ideas in physics: An example from electromagnetic waves
}

\author{
Noah S. Podolefsky and Noah D. Finkelstein \\ Department of Physics, University of Colorado at Boulder, Boulder, Colorado 80309, USA
}

(Received 4 December 2006; published 15 June 2007)

\begin{abstract}
This paper describes a model of analogy, analogical scaffolding, which explains present and prior results of student learning with analogies. We build on prior models of representation, blending, and layering of ideas. Extending this model's explanatory power, we propose ways in which the model can be applied to design a curriculum directed at teaching abstract ideas in physics using multiple, layered analogies. We report on a recent empirical study that motivates this model. Students taught about electromagnetic waves in a curriculum that builds on the model of analogical scaffolding posted substantially greater gains pre- to postinstruction than students taught using a more traditional (non-analogy-based) tutorial (21\% vs $7 \%$ ).
\end{abstract}

DOI: 10.1103/PhysRevSTPER.3.010109

PACS number(s): 01.40.Fk

\section{INTRODUCTION}

Analogies are ubiquitous in both the practice and the teaching of physics. There are many historical examples, from Rutherford's planetary model of the atom ${ }^{1}$ to Maxwell's application of fluid theory to electromagnetism. ${ }^{2}$ Analogies are commonly used to teach physics in lectures and in textbooks. ${ }^{3}$ Because analogies are so common in physics education, there is clearly a need to understand how analogies work, as well as when and why analogies are productive or not for learning physics. Researchers have proposed models of analogy, ${ }^{4-7}$ which have advanced our theoretical understanding, but we argue that these models are insufficient to explain students' use of analogy in practice. Teaching with analogy has been shown to be sometimes productive for learning, but sometimes not, and the reasons for this inconsistency are not well understood. Spiro et al. ${ }^{8}$ suggest teaching with multiple analogies in order to circumvent the drawbacks of single analogies (e.g., single analogies may be misleading or incomplete), especially when teaching complex and difficult topics. Broadly, our efforts build on prior work in order to better understand how, when, and why analogies can be used productively to teach physics, particularly in the context of using multiple analogies. Our prior work revealed implications for the productive use of analogy and called for a new explanatory model. ${ }^{9}$ We extend other researchers' efforts to study mechanisms of analogy and general features of teaching with analogy that lead to their productive use for learning physics. This paper presents a model of analogy, analogical scaffolding, which extends these prior efforts and examines sample data supporting this model.

Our research efforts address the need for further study of teaching with analogy in three ways. (1) The existing literature reports mixed success at teaching with analogy (e.g., Refs. 6,10). Existing models of analogy suggest general approaches to, but do not specifically inform, curriculum design. We make progress toward understanding successful cases, describe specific mechanisms by which analogies can be used productively to teach, and propose a model with direct implications for the design of learning materials using analogies. (2) Often, no single analogy is sufficient for teaching a new idea in physics, especially if that new idea is highly abstract. We propose a mechanism of student use of analogies to learn abstract ideas in physics, specifically by using multiple, layered analogies. (3) We believe models of student learning in physics should begin to explain the complex dynamics of student thinking, allowing for on-the-fly variations in students' thinking that depend crucially on salient factors in the environment. Our model proposes one such mechanism of student reasoning, focusing on how multiple analogies and representations used to teach those analogies interact to influence student thinking.

In this paper, we broaden the scope from a strict definition of analogy to consider the idea of domain comparisons, of which analogy, ${ }^{4}$ metaphor, ${ }^{11}$ and conceptual blends ${ }^{12}$ are particular cases. In all of these cases, mappings of object attributes and relations, as well as other types of connections, are made between two (or more) domains. In the literature dealing with domain comparisons, a domain is often represented by a mathematical set, and a comparison between domains is represented by a mapping, isomorphism, union or another related operation on the two sets. We draw on a range of prior work, focusing on Fauconnier and Turner's theory of conceptual blending, ${ }^{12}$ to develop the analogical scaffolding model. As we will demonstrate, blending includes several features that extend traditional models of analogy. For instance, contrasted with traditional "two-domain" models of analogy, blending is a "multidomain" model, making it a promising model for dealing with multiple analogies. According to Turner and Fauconnier, allowing for multiple domains "introduces a higher degree of variability and a loss of parsimony, but with a corresponding increase in sensitivity and generality. The two-domain model is in fact a special case of the many-space model." 13,14 At the same time we build on work in semiotics ${ }^{15}$ to frame meaning making with representations, and on the work on layering meaning ${ }^{16}$ to see how analogies can scaffold one another. More details on these prior theoretical frameworks will be provided in the next section. Following this section, we briefly describe a selection of the empirical work on which we base our model of analogical scaffolding. Next, we describe this model and address some outstanding questions on teaching with analogies. We explore the utility of this model by applying the model to explain sample data. Finally, we suggest future 
studies and curriculum design which lead naturally from an application of this model.

\section{PREVIOUS ANALOGY THEORY}

A prevailing view of analogy in physics is due to Gentner. ${ }^{4,5}$ Her model, referred to as structure mapping, defines an analogy as a mapping from a base domain to a target domain. Gentner defines attributes of (e.g., color) and relations between (e.g., revolves around) objects, and proceeds to define an analogy as a domain comparison in which, predominantly, relations are mapped rather than attributes. For example, in Rutherford's planetary model of the atom, the solar system is the base domain and the atom is the target domain. The sun, an object, corresponds to the nucleus, the planets correspond to the electrons, and the relationship planets revolve around the sun maps to electrons revolve around the nucleus. There are other possible mappings as well, for instance, the color attributes of the sun and planets could map to the associated components of the atom. According to Gentner's definition of analogy, these attributes do not generally map. Instead, Gentner defines a literal similarity, in which attributes, rather than relations, predominantly map from base to target. An example is the literal similarity between our solar system and another planetary system somewhere else in the galaxy. This delineation between analogy and literal similarity is consistent with expert thinking, but we argue that it may not describe student thinking about analogies. Indeed, when presented with an analogy (as defined by an expert), students may consider attributes equally (or more) valid to map from base to target compared to relations.

Gentner and Gentner ${ }^{10}$ showed empirically that student reasoning about electric circuits was associated with analogies that were generated by the students. Students who used a water analogy out-performed students who used a moving objects analogy on questions about batteries, while students who used a moving objects analogy out-performed students who used a water analogy on questions about resistors. However, in a second experiment, the explicit teaching of water or moving object analogies to students was not found to be as effective as when the students created the analogies themselves. Explanations for this mixed success in teaching with analogies include inadequate student understanding of the base domain, a failure to use the analogy correctly, or a failure to use the analogy at all.

In order to overcome difficulties with teaching analogies, Brown and Clement ${ }^{6}$ suggest bridging as a method to foster productive analogy use. A bridging analogy provides one or more intermediate steps between the base and target intended to help students connect the two. The use of bridging analogies has had mixed success. ${ }^{6,17}$ Researchers attribute student difficulties to the use of analogical comparisons that were too abstract and therefore difficult for students to apply productively. Other studies ${ }^{18,19}$ have confirmed the difficulty in fostering spontaneous analogy generation and productive use by students.

Existing models of analogy have difficulty explaining why analogies are only sometimes successful for affecting student reasoning. In particular, it is not well understood how one knows to make some mappings in an analogy and not others. For instance, in the Rutherford analogy, the attribute "yellow" does not map to the nucleus, whereas "massive" does. Structure mapping ${ }^{4,5}$ proposes the systematicity principle, according to which the mappings selected tend to be those which fit into abstract "higher-order relations," or relations between relations. For instance, in the statement "sun is more massive than planet causes planet revolves around sun," the higher-order relation is cause. It follows that, since "yellow" is not a part of this higher-order relation describing a "central force system," it is less likely to map. However, this mechanism only makes sense if we assume the person using the analogy holds this higher-order relation in mind. It is well known that student knowledge can be fractured, consisting of unstable bits and pieces rather than stable and coherent conceptions or structures. ${ }^{20-22}$ Thus, it is likely that, while in the process of using an analogy to learn something new, students may not be able to apply or even to possess the higher-order relations necessary to use an analogy productively. Indeed, abstractions, such as "central force system," may not exist for students prior to using an analogy. It follows that, if students do not possess these abstract structures, it is unlikely that these students are aware of the relations to map from one structure to another. On the other hand, students may map surface features, such as shape or color, if these features are salient in the presentation of an analogy. One possible explanation for how people overcome this difficulty in using relations productively, also known as the bootstrapping problem, ${ }^{23}$ is that abstractions emerge during the use of an analogy, being produced by the comparison of two domains. Our model builds on this mechanism of comparison in order to make productive use of salient information, leading students to apply increasingly abstract frameworks to learning new ideas. Our claim is that surface features may be productively used in an analogy, especially surface features of the representations presented when teaching with analogy. Therefore, the issue at hand is how students know how and when to use surface features (as well as more abstract relationships) productively and the role of representations in the process of using an analogy.

To be sure, the comparison process depends on a range of mechanisms. Focusing on one possible mechanism, we examine representations. Several lines of research have recently found that student reasoning, whether comparing everyday objects, ${ }^{24}$ learning complex systems by analogy, ${ }^{25}$ learning abstract mathematical principles, ${ }^{26}$ or solving physics problems, ${ }^{27,28}$ can depend strongly on the representational forms presented to students in these activities. These findings were, in some sense, foreshadowed by Chi, Feltovich, and Glaser, ${ }^{22}$ who demonstrated that representations can play a significant role in the way physics experts and novices differently categorize physics problems. In their studies, novices tended to focus on surface features (e.g., problems with inclined planes), while experts focused on physics principles (e.g., conservation of energy) in their grouping of physics problems. Along these lines, other studies have found students may interpret some representations as objects even when the representations stand for something abstract (e.g., interpreting the arrows on electric field lines as sig- 
nifying paths of motion). ${ }^{29-31}$ Elby $^{32}$ terms these sorts of objectlike interpretations What-You-See-Is-What-You-Get (WYSIWYG). Notably, students commonly invoke such objectlike interpretations of electric field ${ }^{33}$ and electromagnetic (EM) wave diagrams. ${ }^{34}$

While such findings may be troubling, these interpretations may also be resources. ${ }^{21}$ Our model builds on this idea, scaffolding learning of abstract ideas by focusing students' attention on surface level thinking that is productive, and using multiple analogies as stepping stones toward more deeply structured abstract reasoning. To this end, the consequences of using representations of varying abstractness are pivotal to our model.

\section{PRIOR RESULTS}

In an ongoing effort to better understand teaching with analogies in physics, we have collected quantitative (e.g., pre vs post assessments) and qualitative (student interviews and classroom observations) data in a series of studies in several large-scale, calculus-based introductory physics courses. This prior work, described briefly here and in detail elsewhere, ${ }^{9}$ demonstrated that analogies can generate inferences when taught to students in a large-scale, calculusbased introductory physics course. In particular, we found that different analogies (e.g., waves on a string or sound waves) were productive for teaching different associated features of EM waves (e.g., traveling waves or plane waves, respectively).

In the first part of this prior study, students were divided into three groups, and each group completed a different tutorial on EM waves during a 50 minute recitation. Drawing on Tutorials in Introductory Physics, ${ }^{35}$ our tutorials used different analogies to teach about EM waves, but all three covered similar content. The first group was taught about waves on a string as preparation for learning about EM waves. The second group was taught about sound waves as preparation, and the third group was not taught an analogy (they were taught only about EM waves). Thus, the target in the first two analogy cases was EM waves, while the base was either string or sound. Students were given a post-test on EM waves, and we found that the distracters that students chose were associated with which analogy they were taught. In the second part of this study, students were divided into two groups and then given a wave representation assessment that focused on waves on a string or sound waves, depending on the treatment group. Students in each group were asked to choose the representation of the string or sound wave that made the most sense to them and explain why, and then answer a follow-up question about the motion of a dot on a string or an air particle in front of a loudspeaker. We found that for both string and sound, the representations students chose were associated with different reasoning about either strings or sound, but the association was substantially stronger for sound. For sound, we also found a strong association between the representation chosen and the answer given to a concept question about the motion of a particle in the path of a sound wave. For instance, students who chose a sine wave representation of sound tended to answer that the particle will move up-down (a transverse wave), while students who chose a picture of compressed and rarefied air particles tended to answer that the particle will move left-right (a longitudinal wave). However, there was no such association between representation and answer for string. Nearly all students answered a similar question about the motion of a dot on a string correctly regardless of their choice of representation.

The results of this study demonstrate two key findings. (1) String and sound analogies generate inferences about EM waves when taught in a large-scale introductory physics course, and the particular inferences generated depend on which analogy students are taught. (2) Representations couple to associated student reasoning, but this coupling is more pronounced for abstract ideas (i.e., sound waves) than for concrete ideas (i.e., waves on a string).

Note that the traveling and plane wave features, both characteristics of EM waves, are individually key elements of string and sound waves, respectively. The study described above revealed that students applied one or the other of these features to EM waves depending on which analogy was taught. We hypothesized, therefore, that the optimal way to teach EM waves would be via both string and sound analogies. This hypothesis is compelling, but not self-evident, ${ }^{36}$ hence motivating the analogical scaffolding model described in the next section.

\section{ANALOGICAL SCAFFOLDING}

\section{Sign, referent, schema}

Our previous large-scale findings ${ }^{9}$ suggested that, for these students, iconographic representations are coupled to associated ideas about physical phenomena (i.e., sound waves). In addition to the study described above, ${ }^{9}$ the development of our model draws on a range of data collected in ongoing large-scale studies as well as numerous interviews conducted with individual students. We limit the scope of this paper to a detailed description of the model and include sample data demonstrating a broad application of the model to curriculum design. More detailed empirical findings which support the model are the subject of a companion paper. ${ }^{37}$

We begin a description of our model with a theoretical account of these findings on the role of representation in an analogy. ${ }^{9}$ We draw on the work of Roth and Bowen ${ }^{15}$ to describe the relationship between a signifier, sign, the thing the sign refers to, referent, and a knowledge structure mediating the sign-referent relationship, schema. The signreferent-schema relationship is represented by the diagram in Fig. 1. We use the word sign to refer to external representations, such as text, graphs, equations, pictures, gestures, or utterances. ${ }^{38}$ According to Rumelhart (Ref. 39, p. 37), "the central function of schemata is in the construction of an interpretation of an event, object, or situation." Thus, schemata (plural of schema) can be considered knowledge structures or resources $^{21}$ employed, among other things, to interpret signreferent relationships. The sign, referent, and schema are represented by nodes at the vertices of the triangle in Fig. 1, and the sides of the triangle represent connections between these three elements. We demonstrate the utility of the representa- 


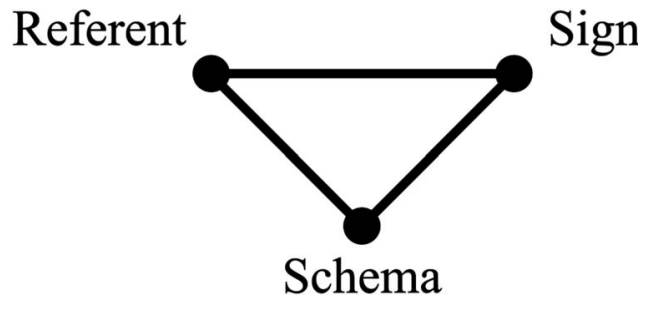

FIG. 1. Relationship between sign, referent, and schema.

tion in Fig. 1 by applying it to the canonical Rutherford analogy.

In Fig. 2(a), the referent is the solar system and the sign is the iconic representation at the upper right vertex. This sign is associated with a particular schema for the solar systemspherical planets orbiting a yellow sun. A subset of the associated schema elements is shown at the lower vertex. Note that a different sign could be associated with a different schema for the same referent. For instance, depicting one of the orbiting objects in Fig. 2(a) as yellow and the central object black would be associated with the sun orbiting the earth. Figure 2(b) shows a similar diagram for the atom. The sign used in Fig. 2(b) cues certain analogical mappings from the solar system which are inherited by the atom schema. This cuing is similar to signs cuing reasoning strategies in the study described above, ${ }^{9}$ where different iconographic representations of sound were associated with different reasoning about the motion of a dust particle. The form of the sign can imply which schema elements to map, i.e., "revolves around," and which elements not to map, i.e., "yellow." Thus far, our model is consistent with the idea that an analogy is a mapping from base (solar system) to target (atom), but we go further and hypothesize that particular signs promote the selection of particular mappings. We also note that signs are not the only factors that can promote mappings.

A consequence of this hypothesis is that subtle changes to the sign can produce significant changes in the use of an analogy. For instance, if the large central sphere in Fig. 2(b) were yellow, this would support, rather than inhibit, the inference that the nucleus is, in fact, yellow. On the other hand, if the sign in Fig. 2(b) were a picture of an electron cloud, the analogy to the solar system might not be cued at all. We suggest that signs are key mechanisms by which analogies can be used productively, and are therefore a key part of teaching with analogy.

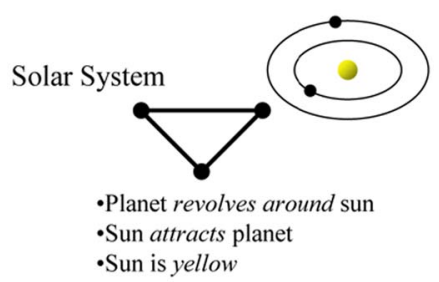

a.

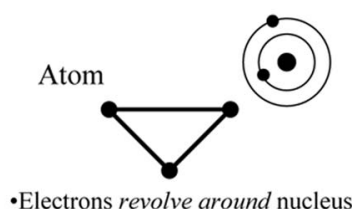

-Nucleus attracts electrons

b.
FIG. 2. (Color) Sign-referent-schema for (a) the solar system and (b) the atom.

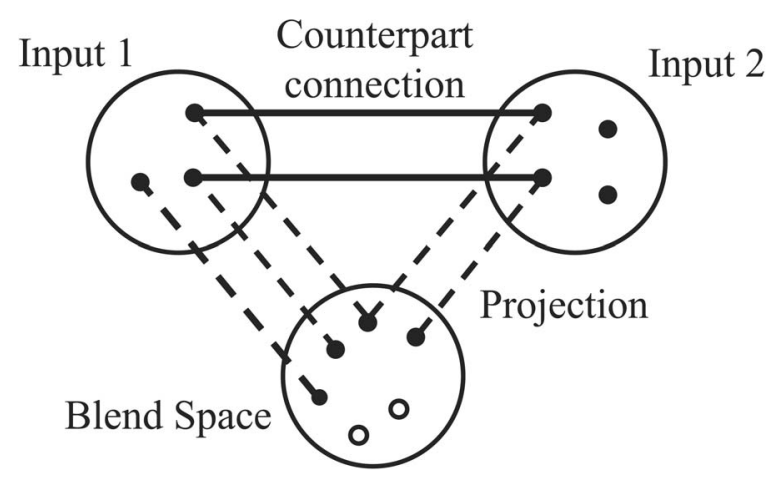

FIG. 3. Conceptual blending diagram. Adapted from Ref. 12.

\section{Conceptual blending}

The structure mapping approach to analogy relies on students' prior knowledge of the base domain, but skirts the issue of students' prior knowledge of the target. ${ }^{6}$ Turning again to the Rutherford analogy, it is likely that students learning about the atom will already have some preconceived ideas about atoms. Further, students may not possess a fully fledged model of the solar system to use as a base. Consider the hypothetical situation in which students' prior knowledge of atoms includes the idea that atoms are made of electrons and a nucleus, but with a number of possible arrangements besides the one given by the Rutherford model. ${ }^{40}$ In this case, students using the solar system analogy are asked to compare the solar system and the atom, in so far as they understand each, and formulate a new conception of the atom based on this comparison.

Conceptual blending ${ }^{12}$ provides a theoretical framework for describing such a process. In a conceptual blend, two input spaces are combined to produce a blend space. This process is represented schematically in Fig. 3. Input and blend spaces are instances of mental spaces, defined by Fauconnier and Turner as "a small conceptual packet constructed as we think and talk, for purposes of local understanding and action." (Ref. 12, p. 102). Elements in one input space (top left of Fig. 3) have counterparts in the other input space (top right of Fig. 3) and elements from each input space can be projected to the blend space (bottom of Fig. 3). Projection is selective, and not all elements are necessarily projected from the inputs to the blend. Connections between input space elements are called vital relations. There are many vital relations-two examples are space and representation. For instance, the very different scales of the solar system and atom are connected by the vital relation space. Electron paths and concentric circles are connected by the vital relation representation. ${ }^{41}$ Blending includes some mechanisms from traditional theories of analogy, such as connections between spaces. ${ }^{13}$ However, an important distinction is that rather than enriching a target domain by mapping elements from a base, in a blend, elements from two input spaces project to a new mental space. ${ }^{13}$ Importantly, input space elements combine in a blend such that, for instance, electron paths and concentric circles are no longer separate, but become orbitals in the blend space. Finally, blends can have emergent structure not contained in the inputs, represented 


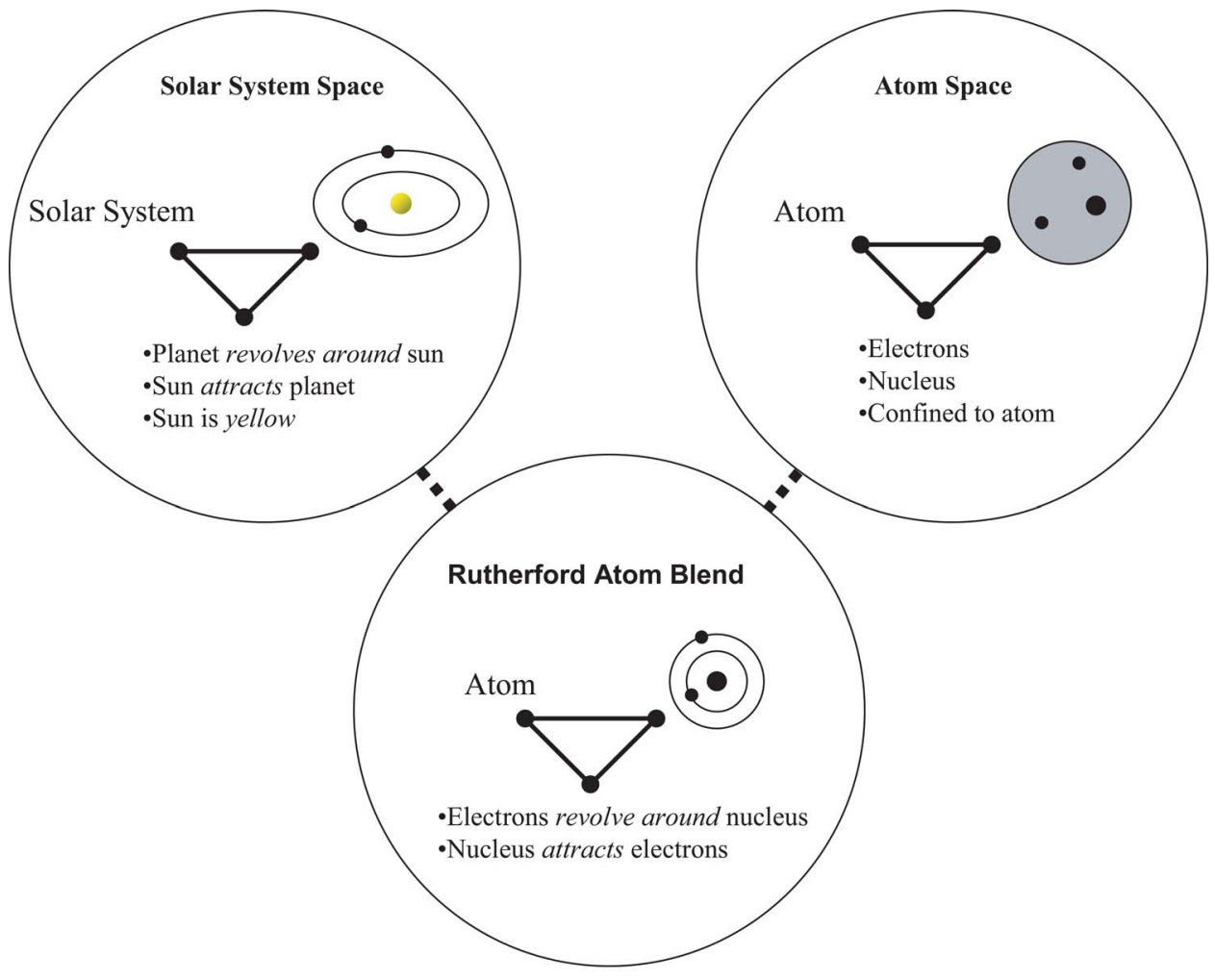

FIG. 4. (Color) Blending the solar system and a rudimentary model of the atom. by the empty circles which appear in Fig. 3. For instance, electrons orbit the nucleus at fixed radii.

Input spaces to a blend can provide structures, or organizing frames, which dictate the way elements in a mental space relate to one another. Different types of blends can be categorized according to whether the organizing frame for the blend comes from one input or from both inputs. When the organizing frame comes from one input, say input 1, the content in input 2 is organized in the blend by the frame from input 1. For instance, in the Rutherford analogy, the solar system space provides a frame that is applied to content from the atom space. ${ }^{42}$ In the case where both inputs contribute frames, the frames are often clashing and only selected elements of each frame are projected to the blend. For instance, a wave on a string evokes a transverse wave frame, while a sound wave evokes a longitudinal wave frame. Blending these clashing frames can produce a blend wherein a generic wave frame contains common elements such as amplitude, frequency, and wavelength. Alternatively, these frames may clash and not be resolved. Organizing frames are somewhat related to relational structures in structure mapping, but, in some cases, students may not bear these frames in mind. Selecting and layering a series of blends establishes the scaffolding around which students may build particular (and generally effective) frames. In the next section, we discuss mechanisms of selection and layering.

\section{Application to the atom}

Conceptual blending can be applied to understand the Rutherford analogy. In our model, we represent each mental space with a sign-schema-referent diagram. Figure 4 shows one input space containing the solar system and associated sign and schema, another input space containing a not fully developed conception of the atom (e.g., a small material object that has protons and electrons) with associated sign and schema, and the blend space containing the Rutherford model of the atom. ${ }^{43}$ At the level of input spaces, the atom schema has not yet inherited the frame from the solar system and could consist of any number of variants. In the blend, selected elements from the atom input space (i.e., electrons and nucleus) and from the solar system input space (i.e., $x$ revolves around $y$ ) are projected to the blend. Put another way, elements from the atom space are organized in the blend by inheriting the solar system frame.

There may be several mechanisms responsible for the selection of elements from each input space. One mechanism may be competition between schemata where the schema that is more tightly coupled to sign and referent (discussed below) is projected with higher probability. Here, the schema associated with the solar system is strongly tied to both the referent and the sign, whereas the input level schema for the atom is not strongly tied to either referent or sign. This would be the case if a student were learning about the atom for the first time. In the blend, the input level schema for the atom is easily discarded in favor of the more tightly coupled solar system schema. This mechanism may apply to individual schema elements rather than entire schemata. Note that unlike the high-order structure hypothesis, this mechanism does not require stable, large-scale structures, but instead relies on the strength of coupling between smaller-scale schema elements and signs. For example, our model predicts that since "revolves around" is tightly coupled to the concentric circles sign, this schema element likely projects to the blend. The nature of this coupling is discussed in more detail in the next section. 
Another mechanism of selection may be the sign used to cue the blend. In Fig. 4, the sign in the blend space is itself a blend of the signs from the input spaces. Its form is such that it can cue the projection of nucleus and electrons from the atom, but also includes the idea of planetary orbits from the solar system. According to Fauconnier and Turner's model, this cuing is a result of the vital relation representation. The first mechanism described above explains how students might create blends based on their prior knowledge. The second mechanism explains how blends can be cued via the use of carefully chosen signs. This second mechanism is particularly relevant to creating learning materials that rely on promoting certain blends.

Blending theory provides an alternative and productive way of describing the Rutherford analogy. However, blending also describes cognitive processes that structure mapping does not. First, rather than a unidirectional mapping from one structure to another, a blend combines inputs from two input spaces to produce a new blend space. Second, blend spaces can become input spaces to new blends. Thus, a blend can be fractal in nature, itself the product of many input spaces, which are themselves blends of several input spaces, and so on. ${ }^{44}$ Lakoff and Nunez ${ }^{16}$ refer to this process of creating more and more complex blends as layering. For instance, one possible input space to the Rutherford blend is the plum pudding model due to J. J. Thompson. Long prior to Thompson, the prevailing model of the atom was an indivisible hard sphere. The progression of atomic models from hard sphere to plum pudding to solar system to electron cloud (quantum) could be viewed as a progression of layered blends. ${ }^{45}$

In the prior example, blends were layered by changing simultaneously the sign and the schema for a given referent (i.e., the atom), wherein a new sign cued a new schema. We could also imagine holding the referent and sign fixed while changing the schema by adding or subtracting schema elements. ${ }^{46}$ For instance, the solar system input space projects a schema which is applied to the atom in Fig. 4. Bohr's contribution of quantized energy could be added to this schema, still represented by the same sign. This addition produces a new blend, with the atom blend from Fig. 4 becoming an input space and Bohr's quantization of energy ${ }^{47}$ providing a second input space. We now say that, unlike planets orbiting the sun, the orbits of electrons implied by Fig. 4 are at fixed radii. In this fashion, the schemata now direct the meaning of the sign, whereas previously, the sign was directing the schemata or elements of the schemata to be used.

Note that while the spatial relationship between the nucleus and electrons is represented explicitly in Fig. 4, this sign does not carry any explicit information about quantization. The spatial relationship can be read out from the sign directly, but the quantization must be learned through a layering process. We represent the result of this layering process by adding intermediate nodes (which represent prior blends) between the sign and schema, shown in Fig. 5. One could imagine any number of intermediate nodes, or blended layers. Each of these layers comprises a blend with an input space wherein a sign is associated with a particular schema for a given referent. For instance, quantization could be rep-

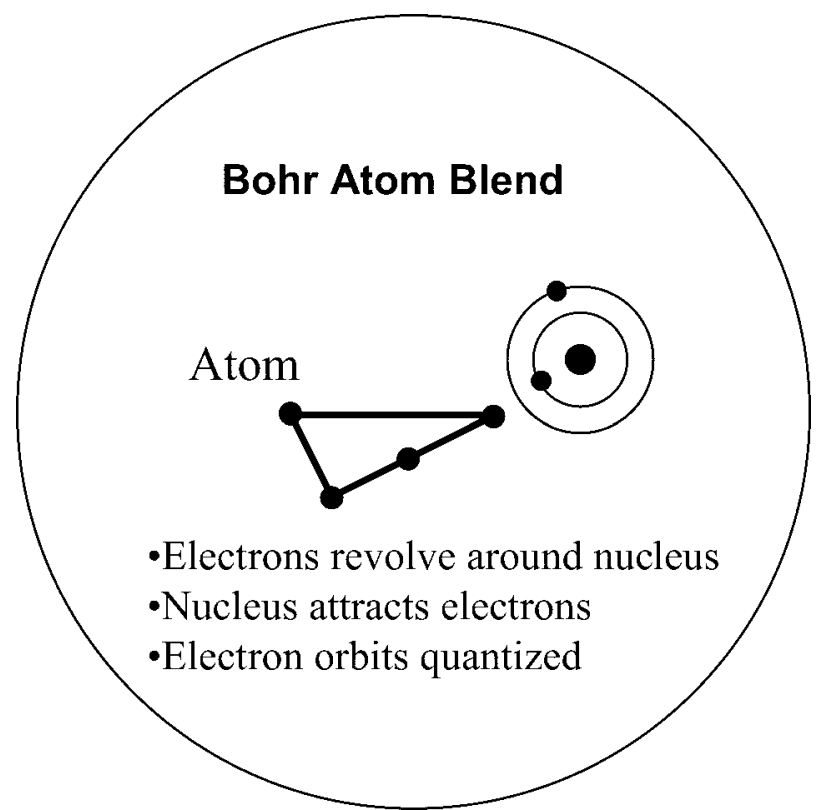

FIG. 5. Adding quantization produces the Bohr atom blend. The additional node between sign and schema represents a blend which incorporates quantization into the schema linked to this sign.

resented by a chart of atomic energy transitions for the hydrogen atom. In the blend, the atom inherits the quantization schema, represented implicitly by the sign in Fig. 5 . We note that these intermediate steps may be along the lines of bridging analogies, building on the ideas of Brown and Clement. ${ }^{6}$

\section{Utility of the concrete and abstract}

Physics ideas are often described as concrete or abstract (e.g., an EM wave is considered highly abstract ${ }^{34}$ ), but the definitions of these descriptors are almost always implicit. Some weigh concrete vs abstract by the degree to which ideas are tied to particular contexts or objects (e.g., "electron" is more concrete than "particle," depending upon an individual's prior knowledge). ${ }^{48}$ Our model allows a more precise definition of concrete and abstract to be encoded in a representation such as Fig. 5. Accordingly, concrete is characterized by a sign-schema-referent triangle with few or no intermediate nodes, while abstract is characterized by a signschema-referent triangle with many intermediate nodes. Since these nodes correspond to blends, more abstract ideas consist of many blended layers. ${ }^{49}$ We will see that EM waves, a highly abstract idea in physics, can be taught via a series of layered blends from concrete to increasingly abstract input spaces.

The fact that the sign, referent, and schema of concrete ideas are more tightly coupled can be extremely productive. We draw on Elby's use of WYSIWYG, What-You-See-Is-What-You-Get. ${ }^{32}$ WYSIWYG is one type of readout strategy, or sign-schema connection, along the lines of $x$ means $x$. For instance, when viewing a graph which is shaped like a hill, a student applying WYSIWYG would think the graph represented a real hill. ${ }^{32}$ Such an interpretation is sometimes productive (e.g., for a graph of height vs 


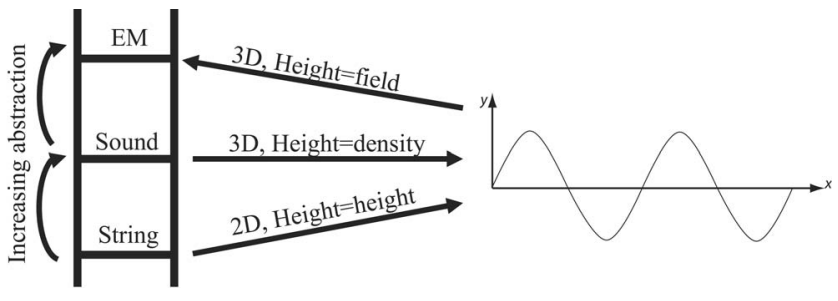

FIG. 6. Scaffolding analogies of increasing abstraction.

distance), and sometimes not (e.g., for a graph of horizontal velocity vs time). We suggest that WYSIWYG, applied to concrete ideas, can be productive for cuing schemata that we would like students to apply to more abstract ideas. For instance, suppose we use a sine wave to represent a wave on a string. Applying WYSIWYG to the sine wave results in a two-dimensional (2D) transverse wave schema, which is correct for a wave on a string. The utility of the concrete is that signs are likely to cue schemata that are productive for learning about referents. In a complementary way, there is utility in using abstract signs such as a sine wave. When representing a wave on a string, this sign (the sine wave) comes to stand for a $2 \mathrm{D}$ transverse wave. If this sine wave is then used to describe an EM wave, "transverse" is cued by the sign and inherited by the EM wave schema. Notably, a pitfall of the abstract is that signs are more likely to cue schemata that are not productive for learning about referents. For instance, 2D may also be inherited by the EM wave schema, in which case another intermediate layer becomes necessary, since EM waves are 3D.

\section{Application to EM waves}

We tie the use of signs, blending, and layering together and apply our model to teaching about EM waves. In our model curricular materials scaffold student learning by layering analogies which progress from concrete to abstract, where progress from relatively concrete ideas to more abstract ideas is represented by climbing to higher rungs of the "ladder" in Fig. 6. ${ }^{48}$ The physics ideas to be learned increase in abstraction as additional blends are layered, compiling more and more elements into a schema connected to a sign, in this case a sine wave. We now describe an analogicalscaffolding-based tutorial on EM waves, wherein students learn about increasingly abstract wave phenomena: waves on a string, followed by sound waves and finally EM waves.

A wave on a string is concrete in that applying WYSIWYG to a sine wave results in an appropriate and productive schema for a wave on a string. Students are presented with a sine wave and a picture of an oscillating string, as shown at the topmost input spaces of Fig. 7. Students are asked to compare these two signs. According to the language of our model, we ask students to blend the schemata cued by these two signs. In practice, these two signs are so similar that most students have trouble seeing how they are different, and this works to their advantage. The schema that is cued for the sine wave includes elements such as two dimensional and transverse. With appropriate cuing (via another sign for the string), a traveling wave schema element is blended into the

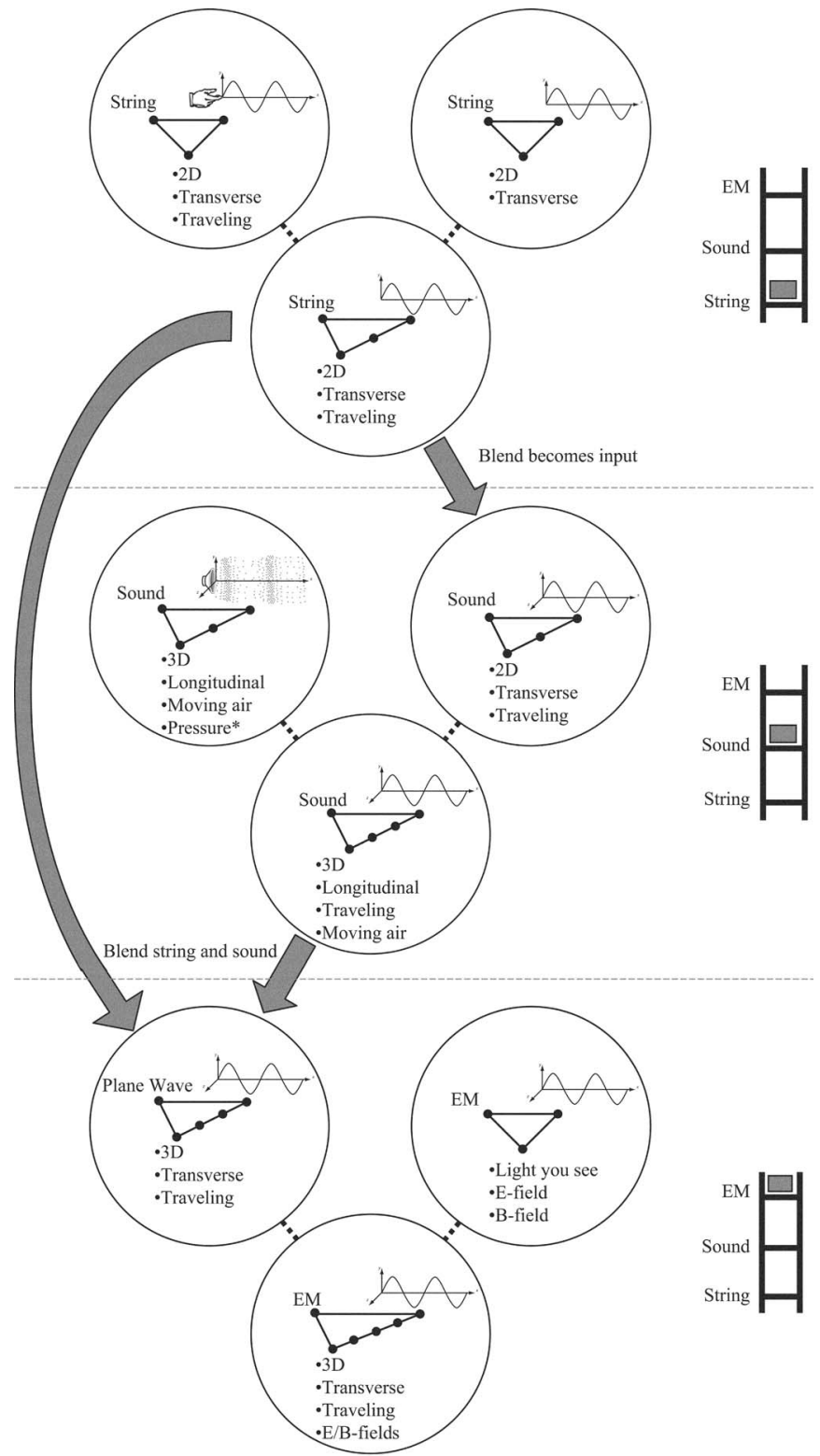

FIG. 7. Layering blends from string (top) to sound (middle) to EM waves (bottom). Each triplet of circles represents two input spaces and a blend space. The string and sound wave blends combine to become an input space for EM waves. Here we show a subset of possible blends. The association of pressure with air density could potentially come from a previous blend.

existing wave on a string schema. That is, a zero crossing is not a node for a traveling wave since a moment later the string will have moved up or down at that $x$ position. The resulting blend is a $2 \mathrm{D}$, transverse, traveling wave schema represented by a sine wave. Students interpret a static sine wave graph as standing for a dynamic, time-varying phenomenon.

Sound is introduced by having students compare a sine wave to an iconographic representation of compressed and rarefied air particles, shown in the second set of input spaces in Fig. 7. WYSIWYG applied to the sine wave does not result in an appropriate schema for sound, but WYISWYG applied to the air particles picture can. Students blend these 
two, and are left with a sine wave that stands for compressed and rarefied air particles, which students associate with pressure (knowledge of which can occur through a blend). Further, since sound is $3 \mathrm{D}$, a peak in the sine wave means high pressure everywhere in a plane with the same $x$ coordinate. In other words, students build up the idea of a plane wave for sound and associate this idea with a sine wave. In the blend, the sine wave stands for a 3D, longitudinal wave that travels in air.

At this point, the sine wave is connected to several schemata, and we use particular cues to compile productive schema elements from a wave on a string and sound waves into a new blend. The resulting schema is represented by a sine wave, and includes the elements transverse, traveling, and 3D. This new blend becomes an input space to the light wave blend. EM waves are introduced, and using a sine wave to represent the EM wave cues the schema just described. Now, the referent is the EM wave, the sign is the sine wave, and the EM wave inherits the schema elements transverse, traveling, and 3D. During this process, more and more layers are blended together, and the result is a highly abstract idea (i.e., an EM wave) represented by a sine wave. According to our model, the sign-schema connection has a large number of intermediate nodes, each corresponding to a blend that is cued for students during the tutorial. If these blends are not made, students will not learn that EM waves are 3D plane waves, as such ideas are only taught in the context of waves on a string and sound waves.

\section{APPLYING ANALOGICAL SCAFFOLDING: AN EXAMPLE STUDY}

We set out to test the utility of our model in a study in which we applied analogical scaffolding to design tutorials to teach EM waves using analogies. This study was conducted in a large-scale, calculus-based introductory physics course - the same course as in the prior study ${ }^{9}$ described above but during a different semester with different students. This course consisted of one lecture section, with three 50 minute lectures per week, and one 50 minute recitation per week with approximately 25 students per recitation section. Students were divided into two groups, denoted as the analogy $(N=72)$ and no-analogy $(N=74)$ groups. (These $N$ include only students who completed all stages of the experiment.) Each group completed a different tutorial during recitation, and all students in a given recitation completed the same tutorial. One treatment group was taught about EM waves using multiple analogies (string and sound), while the other treatment group was taught about EM waves without analogies. Drawing on the original framing of the Tutorials, both versions of the tutorial consisted of three sections. For the analogy group, section 1 covered basic wave concepts such as wavelength, frequency, and amplitude as well as traveling vs standing waves, focusing exclusively on waves on a string. Section 2 covered plane wave concepts, focusing on three-dimensional waves and exclusively on sound waves. Finally, section 3 covered EM wave representations and forces on charges due to the electric and magnetic fields of an EM wave, focusing exclusively on EM waves. The

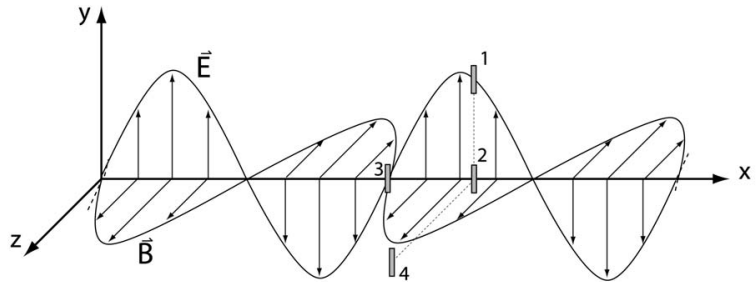

An electromagnetic plane wave propagates to the right in the figure above. Four antennas are labeled 1-4. The antennas are oriented vertically. Antennas 1, 2, and 3 lie in the $x-y$ plane. Antennas 1, 2, and 4 have the same $\mathrm{x}$-coordinate, but antenna 4 is located further out in the $\mathrm{z}$-direction.

Which choice below is the best ranking of the time averaged signals received by each of the antennas. (Hint: the time averaged signal is the signal averaged over several cycles of the wave.)
A) $1=2=3>4$
B) $3>2>1=4$
C) $1=2=4>3$
D) $1=2=3=4$
E) $3>1=2=4$

FIG. 8. EM wave concept question. Correct answer is (D) 1 $=2=3=4$.

no-analogy group used tutorials with the same sections, but always focusing exclusively on EM waves.

Our goals in using analogies to teach EM waves were the following. First, students should learn that for a traveling wave moving in the $+x$ direction, represented by a sine wave, points in the representation where the sine wave crosses the $x$ axis are only nodes at one instant in time. This is a static picture of a dynamic process. For a traveling wave, of which a wave on a string is a concrete and grounded example, points on the wave that are at zero amplitude at one point in time move up and down at later points in time. Second, students should learn that, for a plane wave moving in the $+x$ direction, also represented by a sine wave, points in space that have the same $x$ position have the same amplitude regardless of $y$ or $z$ position. In the analogy treatment, we teach the plane wave idea for sound waves. Finally, students should use both of these ideas applied to EM waves to answer a question like the one shown in Fig. 8.

We compared student learning of EM waves in the analogy and no-analogy groups with a challenging concept question given in lecture on the days immediately prior to and after recitation. This concept question is shown in Fig. 8. Since students from both groups attended the same lecture, students were told not to discuss the question with their inclass peers until after the entire class had finished answering. Individual student responses were collected electronically, and only results from students who attended the recitation and answered both the pre and post concept questions were included in the study. All tutorial interventions took place on the same day, thereby isolating experimental effects of the tutorial treatments.

Answering this question correctly requires students to apply both traveling and plane wave ideas about EM waves. Points 1,2, and 4 are equal since this is a plane wave, and point 3 is equal to the others since this is a traveling wave and we asked for the time-averaged signal. Averaged over several cycles, all four points receive the same signal. Therefore, the correct answer is (D) $1=2=3=4$.

The results for the pre-post EM wave concept question are shown in Fig. 9. The vertical axis shows the shift in 


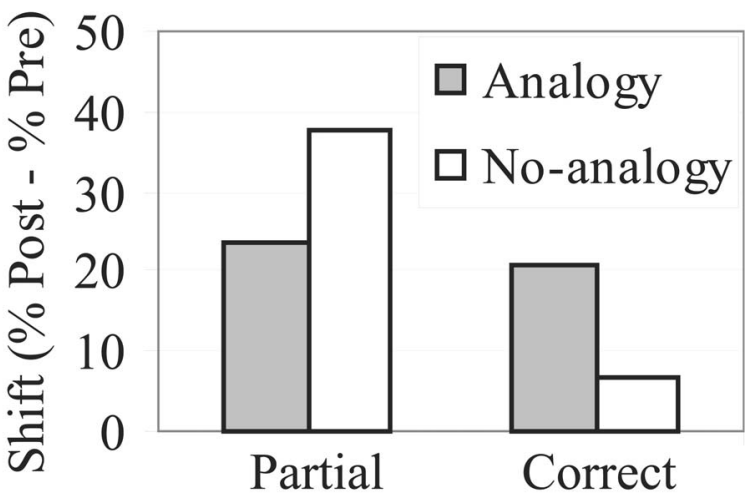

FIG. 9. Results from study 2. Shifts on partially correct answer (Partial) which includes the 3D feature of EM plane waves. The correct answer includes both 3D and traveling wave features.

student responses from before recitation (pre) to after recitation (post) for the analogy and no-analogy groups. The analogy and no-analogy groups were matched on the pretest $\left(\chi^{2}, p>0.2\right)$. The two answers with positive shifts were the correct answer $(1=2=3=4)$ and the main distracter $(1=2$ $=4>3$ ). We consider the main distracter to be partially correct, since it contains the plane wave feature $(1=2=4)$ but not the traveling wave feature ( 3 is equal to 1,2 , and 4 ). In both groups, more than $86 \%$ of students chose one of these two answers on the post concept question. The shift on the correct answer was $21 \%$ in the analogy group and $7 \%$ in the no-analogy group. The shift for the analogy group is statistically significant (McNemar's test, $p=0.01$ ), while the shift for the no-analogy group is not (McNemar's test, $p=0.47$ ). The shift on the partially correct answer was $24 \%$ in the analogy group and $38 \%$ in the no-analogy group. Both of these shifts are statistically significant (McNemar's test, $p$ $<0.01)$. We also examine how students shifted between the correct and partially correct answers from pre to post. We observe that students in the analogy group who answered partially correct on the pretest took the next step to the correct answer four times as often as students in the no-analogy group, while students in the no-analogy group went backward from correct to partially correct at twice the rate of students in the analogy group $\left(\chi^{2}, p=0.01\right)$.

Thus, we find that while students in both groups learned some features of EM waves, students taught with analogies learned substantially more about EM waves than students taught without analogies. Note that the smaller shift to the partially correct answer in the analogy group is due to these students having a greater shift to the correct answer. What these results show is that approximately equal numbers of students in both groups learned the 3D feature of EM plane waves, but significantly more students in the analogy group learned the traveling wave feature. Further, we find the noanalogy treatment fostered more incorrect ideas for students, whereas the analogy treatment helped students learn more correct ideas, as evidenced by shifts between correct and partially-correct.

In the analogy group, students were taught that sound waves are $3 \mathrm{D}$, but they were not taught explicitly that EM waves are 3D. In the no-analogy group, students were taught explicitly that EM waves are 3D. Since both groups learned this characteristic of EM waves, we conclude that the analogies generated inferences about EM waves equally as well as when these characteristics were taught directly for EM waves. Importantly, the analogies also enhanced learning of other characteristics of EM waves and their representations that were not learned as well when taught directly for EM waves (for instance, interpreting a static picture of a sine wave as a traveling wave, taught explicitly about EM waves in the no-analogy group, but taught in the context of a wave on a string in the analogy group). Further studies of this nature are found in a companion paper. ${ }^{37}$

\section{DISCUSSION}

Traditional views of analogy often rely on relatively stable, coherent, large-scale structures that students draw on in using an analogy. Rather than focusing on these largescale structures, our approach builds a schema for EM waves by blending, piece by piece, string and sound wave features. For instance, students apply the 3D characteristic of sound waves to EM waves, but not the longitudinal characteristic of sound. Instead, the transverse (and traveling) wave features of a wave on a string are applied to EM waves. While the use of blending and layering may be common implicit practice by instructors, the analogical scaffolding model provides a mechanism to be explicit about these processes. By being explicit, we may better understand why particular blends work (in an explanatory sense) and predict which blends will work for students in the future (and ultimately allow for curriculum design). We are interested in how a given target may require multiple base domains, and propose mechanisms that work to compile ideas from multiple analogies. To this end, we build on significant prior work on analogy (e.g., Refs. 4-6) and draw on several existing cognitive models (i.e., blending, ${ }^{12}$ semiotics, ${ }^{15}$ and layering ${ }^{16}$ ) to assemble the analogical scaffolding model.

We demonstrate the utility of analogical scaffolding by applying this model to design tutorials using analogies. Our model explains how signs associate with schemata, affecting the way students blend and project schemata when learning about waves, leading to observed differences between analogy and no-analogy conditions. Note that these signs carry different meaning depending upon treatment. In both treatment groups, students create blends associated with signs, but in this case blends involving analogies are more productive than blends without analogies. According to this model, students can be cued to make productive blends under certain conditions. For instance, by presenting a sign to students that shares surface level features with a schema to project, in this case a concrete picture of an oscillating string, students are cued to project the dynamic oscillations of a traveling wave on a string to an EM wave. The projection is promoted by associating this schema with a sign that is used consistently across analogical domains, cuing this same schema in the target domain. For instance, our model predicts that students who ground the sine wave in the concrete representation of a wave on a string that is traveling would more likely link traveling as a schema element to this sign, a sine wave, than 
students who do not ground the sign with concrete representations. And in fact, students simply told that the sine wave represents traveling (as in the case for the no-analogy group) do not link the sign (sine wave) to the schema element (traveling) for abstract ideas (EM) as often as those in the analogy group. To summarize, our model poses one mechanism of analogy, whereby students make meaning of signs by blending signs and schemata in one domain and apply this meaning to another domain.

In order to apply our model, one can employ diagrams similar to Figs. 4 and 7. We may consider the constituent parts of these diagrams as modules to be assembled in ways appropriate to certain learning goals. There may be an inexhaustible number of ways to assemble these modules (two examples are provided in the Appendix). To determine the form of such a diagram, it may be necessary to work backwards from a desired "target blend." For instance, it may be necessary to "unpack" or "explode" 50 the blend at the bottom of Fig. 7, working upward in the diagram to determine the preceding blends. While this seems a natural activity for teachers designing curriculum, future studies could explore the effect on student learning if the students do the unpacking. A suite of experimental studies are ongoing to delineate the utility of this model at a variety of scales - the macroscopic (across layer to design learning materials) and the microscopic (within layer to understand student sensemaking of particular representations and signs). ${ }^{37}$

\section{CONCLUSION}

Drawing on empirical results as well as previous theoretical frameworks, we have developed a model of analogy, analogical scaffolding. This model is consistent with our experimental findings, and, moreover, our model is also consistent with prior framings of analogy such as mapping and bridging. Within the framework of the model, an analogy can be considered as a mapping from a base domain to a target domain, but we adopt the framework of conceptual blending ${ }^{12}$ to expand this view of analogy to include bidirectional projections as well as multilayered analogies. Bridging analogies can be considered a process of compiling several ideas about a target via a linked set of blended mental spaces. Our model does not require stable and coherent knowledge structures that exist a priori, but allows for smaller-scale schemata to be cued and blended with other schemata on the fly. Finally, by suggesting how schemata can be cued and blended, our model can be directly applied to curriculum design.

A preliminary study, setting the groundwork for our model, demonstrated that students taught with a curriculum based on analogical scaffolding outperformed students taught the same ideas but with a more traditional (non-analogybased) curriculum. The model posits a mechanism explaining why students make effective use of abstract representations in the analogy treatment but not in the no-analogy treatment. Further establishment of this model is the subject of ongoing experimental work. $^{37}$

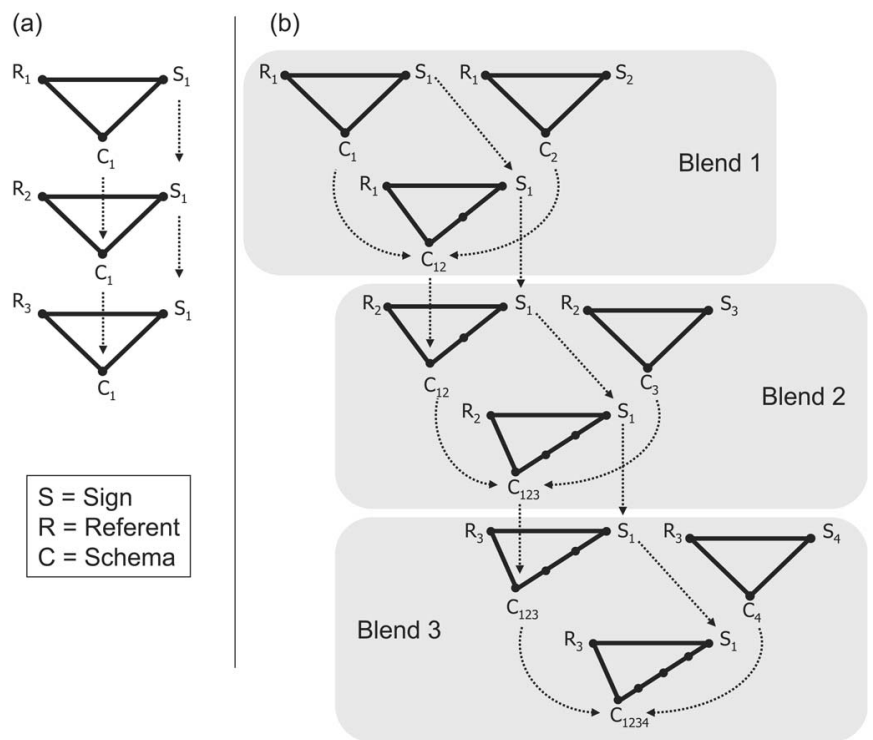

FIG. 10. Two possible diagrams representing layering of mental spaces.

\section{ACKNOWLEDGMENTS}

This work has been supported by the National Science Foundation (Grants No. DUE-CCLI 0410744 and No. REC CAREER 0448176), the AAPT/AIP/APS (Colorado PhysTEC program), and the University of Colorado. We wish to extend sincere thanks to Jamie Nagle, Shijie Zhong, and especially to Edward Redish, Thomas Bing, Michelle Zandieh, Michael Wittmann, and the Physics Education Research (PER) at Colorado Group, particularly Steven Pollock and Wendy Adams, for essential and significant contributions to this work.

\section{APPENDIX}

Figures 10(a) and 10(b) show two possible diagrams representing layering of mental spaces. Sign, referent, and schema are denoted $S, R$, and $C$, respectively. In Fig. 10(a), the same sign $\left(S_{1}\right)$ cues an associated schema $\left(C_{1}\right)$ in each layer, associated with three different referents $\left(R_{1-3}\right)$. In Fig. 10(b), several signs associate with several schemata and referents. Here, schemata are compiled into increasingly complex schemata by way of blending. These schemata come to associate with new referents, followed by further blending. The different blends are connected via a sign that remains constant throughout each blend (in this case, $S_{1}$ ). For example, consider Fig. 10(a) with $S_{1}$ a sine wave, associated with the same schema $\left(C_{1}\right)$ for each referent. This would be initially productive if $R_{1}$ were a wave on a string, but could lead to inappropriate projections if $R_{2}$ were a sound wave. The EM wave example described in this paper is based on Fig. 10(b). We believe this model of representational meaning and analogy can be used to effectively explain others' findings about use of representation, from WYSIWYG (Ref. 32) to useful abstraction in representation. ${ }^{26}$ 
${ }^{1}$ E. Rutherford, The scattering of $\alpha$ and $\beta$ particles by matter and the structure of the atom, Philos. Mag. 6, 21 (1911).

${ }^{2}$ J. C. Maxwell, in The Scientific Papers of James Clerk Maxwell, edited by W. D. Niven (Cambridge University Press, Cambridge, U.K., 1890; Dover, New York, 1952), pp. 367-369.

${ }^{3}$ M. K. Iding, How analogies foster learning from science texts, Instr. Sci. 25, 233 (1997).

${ }^{4}$ D. Gentner, Structure-mapping: A theoretical framework for analogy, Cogn. Sci. 7, 155 (1983).

${ }^{5}$ A. B. Markman and D. Gentner, Structure mapping in the comparison process, Am. J. Psychol. 113, 501 (2000).

${ }^{6}$ D. E. Brown and J. Clement, Overcoming misconceptions via analogical reasoning: Abstract transfer versus explanatory model construction, Instr. Sci. 18, 237 (1989).

${ }^{7}$ K. J. Holyoak and P. Thagard, The analogical mind, Am. Psychol. 52, 35 (1997).

${ }^{8}$ R. J. Spiro, P. J. Feltovich, R. L. Coulson, and D. K. Anderson, in Similarity and Analogical Reasoning, edited by S. Vosniadou and A. Ortony (Cambridge University :Press, Cambridge, U.K., 1989).

${ }^{9}$ N. S. Podolefsky and N. D. Finkelstein, Use of analogy in learning physics: The role of representations, Phys. Rev. ST Phys. Educ. Res. 2, 020101 (2006).

${ }^{10}$ D. Gentner and D. R. Gentner, in Mental Models, edited by D. Gentner and A. Stevens (Lawrence Erlbaum Press, Hillsdale, NJ, 1983).

${ }^{11}$ G. Lakoff, in Metaphor and Thought, 2nd ed., edited by A. Ortony (Cambridge University Press, Cambridge, U.K., 1993).

${ }^{12} \mathrm{G}$. Fauconnier and M. Turner, The Way We Think: Conceptual Blending and the Mind's Hidden Complexities (Basic Books, New York, 2003).

${ }^{13}$ M. Turner and G. Fauconnier, Conceptual integration and formal expression, Metaphor Symbol. Act. 10, 183 (1995).

${ }^{14} \mathrm{G}$. Fauconnier, In The Analogical Mind, edited by D. Gentner, K. J. Holyoak, and B. N. Kokinov (MIT Press, Cambridge, MA, 2001).

${ }^{15}$ W. M. Roth and G. M. Bowen, Complexities of graphical representations during lectures: A phenomenological approach, Learn. Instr. 9, 235 (1999).

${ }^{16} \mathrm{G}$. Lakoff and R. Nunez, Where Mathematics Comes From: How the Embodied Mind Brings Mathematics into Being (Basic Books, New York, 2001).

${ }^{17}$ D. E. Brown, Facilitating conceptual change using analogies and explanatory models, Int. J. Sci. Educ. 16, 201 (1994).

${ }^{18}$ M. L. Gick and K. J. Holyoak, Analogical problem solving, Cogn. Psychol. 12, 306 (1980).

${ }^{19}$ M. L. Gick and K. J. Holyoak, Schema induction and analogical transfer, Cogn. Psychol. 15, 1 (1983).

${ }^{20}$ A. A. diSessa, in Constructivism in the Computer Age, edited by G. Forman and P. B. Pufall (Lawerence Erlbaum, Hillsdale, NJ, 1988).

${ }^{21}$ D. Hammer, A. Elby, R. F. Scherr, and E. F. Redish, in Transfer of Learning from a Modern Multidisciplinary Perspective, edited by J. Mestre (Information Age Publishing, Greenwich, CT, 2005), pp. 89-120.

${ }^{22}$ M. Chi, P. Feltovich, and R. Glaser, Categorization and representation of physics problems by experts and novices, Cogn. Sci. 5, 121 (1981)

${ }^{23}$ K. J. Kurtz, C. Mao, and D. Gentner, Learning by analogical bootstrapping, J. Learn. Sci. 10, 417 (2001).
${ }^{24}$ L. L. Namy and D. Gentner, Making a silk purse out of two sow's ears: Childrens' use of comparison in category learning, J. Exp. Psychol. Gen. 131, 5 (2002).

${ }^{25}$ R. L. Goldstone and Y. Sakamoto, The transfer of abstract principles governing complex adaptive systems, Cogn. Psychol. 46, 414 (2003).

${ }^{26}$ V. M. Sloutsky, J. A. Kaminsky, and A. F. Heckler, The advantage of simple symbols for learning and transfer, Psychon. Bull. Rev. 12, 508 (2005).

${ }^{27}$ P. B. Kohl and N. D. Finkelstein, Student representational competence and self-assessment when solving physics problems, Phys. Rev. ST Phys. Educ. Res. 1, 010104 (2005).

${ }^{28}$ D. E. Meltzer, Relation between students' problem-solving performance and representational mode, Am. J. Phys. 73, 463 (2005).

${ }^{29} \mathrm{We}$ note that this objectlike reasoning may also apply when the sign is in the form of words, and e.g., interpreting the phrase "step function" as describing a stair-step-like object. See, for example, D. T. Brookes and E. Etkina, Do our words really matter? Case Studies From Quantum Mechanics, Proceedings of the 2005 Physics Education Research Conference (AIP, Melville, NY, 2006).

${ }^{30}$ M. Reiner, J. D. Slotta, M. T. H. Chi, and L. B. Resnick, Naïve physics reasoning: A commitment to substance-based conceptions, Cogn. Instruct. 18, 1 (2000).

${ }^{31}$ D. L. Schwartz, Reasoning about the referent of a picture versus reasoning about the picture as the referent: An effect on visual realism, Mem. Cognit. 23, 709 (1995).

${ }^{32}$ A. Elby, What students' learning of representation tells us about constructivism, J. Math. Behav. 19, 481 (2000).

${ }^{33}$ S. Tornkvist, K. A. Pettersson, and G. Transtromer, Confusion by representation: On students' comprehension of the electric field concept, Am. J. Phys. 61, 335 (1993).

${ }^{34}$ B. S. Ambrose, P. R. L. Heron, S. Vokos, and L. C. McDermott, Student understanding of light as an electromagnetic wave: Relating the formalism to physical phenomena, Am. J. Phys. 67, 891 (1999).

${ }^{35}$ L. C. McDermott and P. S. Schaffer, Tutorials in Introductory Physics (Prentice-Hall, Upper Saddle River, NJ, 2001).

${ }^{36}$ Alternatively, one might hypothesize that this approach would lead to more confusion for students. The string and sound analogies may interfere in problematic ways, or there may be simply too much information for students to learn at once.

${ }^{37}$ N. Podolefsky and N. Finkelstein, http://per.colorado.edu/ analogy/index.htm

${ }^{38}$ In common physics parlance, sign and representation share the same meaning. In other sciences, representation often refers to internal or mental representations, along the lines of mental models or schemata.

${ }^{39}$ D. E. Rumelhart, in Theoretical Issues in Reading Comprehension, edited by R. J. Spiro, B. C. Bruce, and W. F. Brewer (Lawrence Erlbaum, Hillsdale, NJ 1980), pp. 35-38.

${ }^{40}$ This was the state of scientific knowledge prior to Rutherford.

${ }^{41}$ M. Turner, Compression and representation, Lang. Lit. 15, 17 (2006).

${ }^{42}$ In the language of blending theory, this is referred to as a "single scope" blend, where the organizing frame of one mental space (solar system) is applied to the elements of another (atom). For more, see Ref. 12.

${ }^{43}$ One might ask why an external representation is contained within a mental space. We side with the view that artifacts of the envi- 
ronment, such as pictures on paper, are key components of cognition, and hence mental spaces. See M. Wilson, Six views of embodied cognition, Psychon. Bull. Rev. 9, 625 (2002).

${ }^{44}$ Each of these sign-schema-referent triangles may be considered a blend, whereby the sign, referent, and schema are connected by vital relations. In the case of the solar system, a picture of the solar system (sign) may be connected to the real solar system (referent) via the vital relation representation. In the Rutherford atom blend, the size of both the solar system and atom referents are scaled to a human scale (the actual size of the sign) via the vital relation space. Role and value vital relations may be involved, whereby the role central object takes the values sun or nucleus, and the role outer object takes the values planet or electron.

${ }^{45}$ In this case, the historical progression happens to match a pedagogy that may be productive. We do not mean to suggest, however, that pedagogy should, in general, follow historical accounts of discovery.

${ }^{46}$ These changes to sign-schema-referent relations may be along the lines of changes to Wittmann's resource graphs. See M. C. Wittmann, Using resource graphs to represent conceptual change, Phys. Rev. ST Phys. Educ. Res. 2, 020105 (2006).

${ }^{47}$ It might be more accurate to say Planck's quantization of energy, which Bohr blended with Rutherford's model.
${ }^{48}$ The delineation of abstract and concrete may depend on the level of expertise. To an expert physicist, an electron is a particular type of particle, and thus the electron is more concrete. To a student, to whom an electron may be an unfamiliar idea, "particle" may be more concrete in the sense of being connected to a real object, like a dust particle. In this sense, students' prior knowledge plays a role in our model to the extent that we can determine which ideas are already concrete for students, and which remain abstract. For a detailed analysis of levels of abstraction, see S. I. Hayakawa, Language in Thought and Action, 3rd ed. (Harcourt Brace Jovanovich, New York, 1972).

${ }^{49}$ Such a notion of abstraction being a series of blends is consistent with Lakoff and Nunez's notion of layering (Ref. 16). The level of perceived abstraction may depend on the student, in that as students become increasingly familiar with abstract sign-schema relations, these relations may become increasingly treated as concrete. In this case, nodes may become so tightly coupled for an expert that the sign-schema link is compressed and the nodes disappear. For instance, to the expert physicist, the notions of "light" and "wave" are not separate ideas-to this expert, light is a wave.

${ }^{50}$ Saalih Allie (private communication). 